\title{
Factors affecting appearance of a normal myocardial perfusion scan
}

\author{
Piotr Slomka, PhD, a and Guido Germano, $\mathrm{PhD}^{\mathrm{a}}$ \\ a Department of Medicine, Cedars-Sinai Medical Center, David Geffen School of Medicine, \\ UCLA, Los Angeles
}

Received Mar 13, 2017; accepted Mar 14, 2017

doi:10.1007/s12350-017-0857-4

\section{See related article, pp. 1645-1654}

\section{INTRODUCTION}

Tools for the relative quantification and automated scoring of myocardial perfusion imaging (MPI) are readily available in the clinic. In most implementations,${ }^{1-4}$ the quantification of the relative hypoperfusion is accomplished by statistical comparisons of the pixels on the polar maps from normal patients. This reference set of normal scans is usually referred to as a "normal database". There have been many studies reporting the polar map count distribution of normal perfusion scans. Differences in the appearance of the normal perfusion have been reported due to multiple technical and patient factors. Clinicians utilizing the quantitative tools should be aware of the possible pitfalls in automated image quantification due to the inappropriate (mismatched) normal perfusion limits.

\section{PATIENT POSITION AND NORMAL PERFUSION APPEARANCE}

In this issue of Journal of Nuclear Cardiology ${ }^{\circledR}$, Kracsko et $\mathrm{al}^{5}$ report the differences between supine and sitting (upright) MPI images. They evaluate 55 patients undergoing two sequential gated ${ }^{99 \mathrm{~m}} \mathrm{Tc}$-sestamibi scans (one sitting, one upright) on two different cameras (similar models from the same vendor). The authors assessed the differences between various quantitative

Reprint requests: Piotr Slomka, PhD, Department of Medicine, CedarsSinai Medical Center, David Geffen School of Medicine, UCLA, Los Angeles; slomkap@cshs.org

J Nucl Cardiol 2018;25:1655-7.

1071-3581/\$34.00

Copyright (C) 2017 American Society of Nuclear Cardiology. parameters obtained from these two scans. They found some differences in both the functional parameters (ventricular volumes and mass) and the quantitative perfusion parameters. In particular, they reported that perfusion defects were in general larger in the upright position as compared to the supine position and that more studies in the upright position were interpreted as abnormal. Based on these findings, the authors recommend the use of the separate normal perfusion databases for the supine and upright positions.

Some of the limitations of the study by Kracsko et al should be highlighted. Patient position during the scan is only one of the potential causes of the differences in MPI measurements. In the study by Kracsko et al, supine images were always performed before upright images, and in fact, different camera systems (although from the same vendor) were used for supine and subsequent upright images. They also used only stress images in their evaluation of differences. It is possible that in addition to the changed patient position, the timing differences between these two stress scans could affect quantitative results. Nevertheless, a small time difference between two stress scans is not likely to be a key factor. In a study of reproducibility with the same camera position, no significant biases were found in the quantitative perfusion parameters in two sequential scans with the same injection. ${ }^{6}$ Perhaps the biggest limitation, however, is that the authors utilized a standard normal database, which was not matched to the camera studied, and they did not evaluate the use of position-specific databases-even though they proposed the use of such normal limits in their conclusions.

Several previous studies analyzed the effect of patient position on myocardial perfusion quantification. In a study by Nakazato et al, supine and upright imaging was studied on the same camera. ${ }^{7}$ They demonstrated that despite small differences, combining the normal limits (using both supine and upright images) did not affect the diagnostic accuracy. In addition, the average 
Table 1. Factors potentially affecting the characteristics of normal perfusion limits

\begin{tabular}{lll}
\hline \multicolumn{1}{c}{ Factors } & Studies & \multicolumn{1}{c}{ Specific limits required } \\
\hline Gender & $9,17,19$ & Yes. Potentially no with attenuation correction \\
Position & $5,7,8,11,12$ & Possibly \\
Scanner type/geometry & 9 & Yes \\
Attenuation correction & $9,17,19$ & Yes \\
High dose/low dose & 10 & Yes \\
Body mass index & 17 & No \\
Ethnicity & $13-16,20$ & Possibly \\
Stress protocol (adenosine/exercise) & 18 & No \\
Tracer ( ${ }^{201}$ Tl vs ${ }^{99 m}$ Tc) & 21 & Yes \\
Tracer (sestamibi vs tetrofosmin) & 22 & Probably no \\
\hline
\end{tabular}

myocardial uptake between supine, upright, and combined (upright/supine) databases did not differ significantly in any of the 17 segments for either men or women. This was true for supine/upright normal limits created for both ${ }^{99 \mathrm{~m}} \mathrm{Tc}$ or ${ }^{201} \mathrm{Tl}$ tracers. Thus, the Nakazato et al study, albeit performed on a different camera, contradicts the conclusions of Kracsko et $\mathrm{al}^{5}$ with regards to the need for the position-specific supine/ upright databases. On the other hand, Nishina et al demonstrated unequivocally that separate supine and prone normal limits are indeed required for the quantification of supine and prone MPI on the conventional dual-head camera system. ${ }^{8}$ It is quite likely that the variability between supine and prone MPI is greater than the variability between supine and upright images due to larger differences in patient position.

This study reminds us of the often-asked questions in nuclear cardiology. How many different databases are needed for quantification of MPI and what factors are key determinants of the normal perfusion appearance on MPI? In the recent review of quantification with normal limits, Rubeaux et al summarized the developments with regards to specific normal limits related to the new equipment and imaging protocols. ${ }^{9}$ The key parameters defining the characteristics of the normal database are the camera type, count level (low-dose vs high-dose affecting the variation of the normals), ${ }^{10}$ attenuation correction (yes/no), patient position (as studied by Kracsko et al), ${ }^{5,7,8,11,12}$ and possibly patient ethnicity. For example, an extensive validation of Japanese-specific databases ${ }^{13-16}$ clearly demonstrated the need for the creation of population-specific databases. On the other hand, it has been shown that normal limits generated for obese patients do not show significant differences as compared to non-obese patients. ${ }^{17}$ Another potential factor is the stress test type (exercise or pharmacological). However, stress protocols-specific databases were studied and did not reveal differences in the quantitative accuracy as compared to mixed databases using both exercise and adenosine normal scans. ${ }^{18}$ In Table 1 , we summarize various possible factors potentially affecting normal perfusion limits, with publications reporting effects of these factors on normal perfusion distribution in SPECT MPI.

\section{CONCLUSIONS}

Automated quantification of MPI has been demonstrated to achieve a very high level of accuracy, rivaling expert observers. Nevertheless, the quantification process is dependent on the selection of appropriate normal perfusion limits. As these automated systems become more commonly used in the clinical practice, aiding the clinical scoring and reporting, it is of great importance to ensure that the appropriate (matched) limits are used to ensure the maximum accuracy. Therefore, it is helpful to know how various factors may affect normal perfusion scan. Patient position during the MPI scan is one of the factors potentially influencing the appearance of the normal perfusion and should be considered when applying automated quantification in clinical practice. The need for position-specific normal limits, however, may depend on the camera geometry and the imaging protocol.

\section{Disclosure}

Cedars-Sinai receives royalties for licensing of quantitative perfusion software, a portion of which is shared with the inventors of which Piotr Slomka and Guido Germano are among.

\section{References}

1. Germano G, Kavanagh PB, Slomka PJ, Van Kriekinge SD, Pollard G, Berman DS. Quantitation in gated perfusion SPECT imaging: The Cedars-Sinai approach. J Nucl Cardiol 2007;14:433-54. 
2. Garcia EV, Faber TL, Cooke CD, Folks RD, Chen J, Santana C. The increasing role of quantification in clinical nuclear cardiology: The Emory approach. J Nucl Cardiol 2007;14:420-32.

3. Ficaro EP, Lee BC, Kritzman JN, Corbett JR. Corridor4DM: The Michigan method for quantitative nuclear cardiology. J Nucl Cardiol 2007; 14:455-65.

4. Liu YH. Quantification of nuclear cardiac images: The Yale approach. J Nucl Cardiol 2007;14:483-91.

5. Kracskó B, Barna S, Sántha O, et al. Effect of patient positioning on the evaluation of myocardial perfusion SPECT. J Nucl Cardiol 2017. doi:10.1007/s12350-017-0865-4.

6. Xu Y, Hayes S, Ali I, et al. Automatic and visual reproducibility of perfusion and function measures for myocardial perfusion SPECT. J Nucl Cardiol 2010;17:1050-7.

7. Nakazato R, Tamarappoo BK, Kang X, et al. Quantitative uprightsupine high-speed SPECT myocardial perfusion imaging for detection of coronary artery disease: Correlation with invasive coronary angiography. J Nucl Med 2010;51:1724-31.

8. Nishina H, Slomka PJ, Abidov A, et al. Combined supine and prone quantitative myocardial perfusion SPECT: Method development and clinical validation in patients with no known coronary artery disease. J Nucl Med 2006;47:51-8.

9. Rubeaux M, Xu Y, Germano G, Berman DS, Slomka PJ. Normal databases for the relative quantification of myocardial perfusion. Curr Cardiovasc Imaging Rep 2016;9:22.

10. Nakazato R, Berman DS, Hayes SW, et al. Myocardial perfusion imaging with a solid-state camera: Simulation of a very low dose imaging protocol. J Nucl Med 2013;54:373-9.

11. Nishiyama Y, Miyagawa M, Kawaguchi N, et al. Combined supine and prone myocardial perfusion single-photon emission computed tomography with a cadmium zinc telluride camera for detection of coronary artery disease. Circ J 2014;78:1169-75.

12. Nakazato R, Slomka PJ, Fish M, et al. Quantitative high-efficiency cadmium-zinc-telluride SPECT with dedicated parallel-hole collimation system in obese patients: Results of a multi-center study. J Nucl Cardiol 2014;22:266-75.

13. Nakajima K. Normal values for nuclear cardiology: Japanese databases for myocardial perfusion, fatty acid and sympathetic imaging and left ventricular function. Ann Nucl Med 2010;24:12535.

14. Nakajima K, Matsuo S, Kawano M, et al. The validity of multicenter common normal database for identifying myocardial ischemia: Japanese Society of Nuclear Medicine Working Group database. Ann Nucl Med 2010;24:99-105.

15. Nakajima K, Okuda K, Kawano M, et al. The importance of population-specific normal database for quantification of myocardial ischemia: Comparison between Japanese 360 and 180degree databases and a US database. J Nucl Cardiol 2009;16:42230.

16. Nakajima K, Matsumoto N, Kasai T, Matsuo S, Kiso K, Okuda K. Normal values and standardization of parameters in nuclear cardiology: Japanese Society of Nuclear Medicine Working Group database. Ann Nucl Med 2016;30:188-99.

17. Slomka PJ, Fish MB, Lorenzo S, et al. Simplified normal limits and automated quantitative assessment for attenuation-corrected myocardial perfusion SPECT. J Nucl Cardiol 2006;13: $642-51$.

18. Slomka PJ, Nishina H, Berman DS, et al. Automated quantification of myocardial perfusion SPECT using simplified normal limits. J Nucl Cardiol 2005;12:66-77.

19. Grossman GB, Garcia EV, Bateman TM, et al. Quantitative Tc-99m sestamibi attenuation-corrected SPECT: Development and multicenter trial validation of myocardial perfusion stress gender-independent normal database in an obese population. J Nucl Cardiol 2004;11:263-72.

20. Li D, Li D, Feng J, Yuan D, Cao K, Chen J. Quantification of myocardial perfusion SPECT studies in Chinese population with Western normal databases. J Nucl Cardiol 2010;17:48693.

21. Matsunari I, Boning G, Ziegler SI, et al. Attenuation-corrected rest thallium-201/stress technetium 99m sestamibi myocardial SPECT in normals. J Nucl Cardiol 1998;5:48-55.

22. Flamen P, Bossuyt A, Franken PR. Technetium-99m-tetrofosmin in dipyridamole-stress myocardial SPECT imaging: Intraindividual comparison with technetium-99m-sestamibi. J Nucl Med 1995;36:2009-15 